\title{
Exploring Green Interference Power for Wireless Information and Energy Transfer in the MISO Downlink
}

\author{
Gan Zheng*, Christos Masouros ${ }^{\dagger}$, Ioannis Krikidis ${ }^{\ddagger}$, Stelios Timotheou ${ }^{\S}$ \\ ${ }^{*}$ School of Computer Science and Electronic Engineering, University of Essex, Colchester, UK, CO4 3SQ \\ $\dagger$ Department of Electronic \& Electrical Engineering, University College London, Torrington Place, London, WC1E 7JE \\ $\ddagger$ Department of Electrical and Computer Engineering, University of Cyprus, Cyprus \\ $\S$ KIOS Research Center for Intelligent Systems and Networks, University of Cyprus, Cyprus \\ Email: ganzheng@essex.ac.uk, chris.masouros@ieee.org, krikidis@ucy.ac.cy, timotheou.stelios@ucy.ac.cy
}

\begin{abstract}
In this paper we propose a power-efficient transfer of information and energy, where we exploit the constructive part of wireless interference as a source of green useful signal power. Rather than suppressing interference as in conventional schemes, we take advantage of constructive interference among users, inherent in the downlink, as a source of both useful information and wireless energy. Specifically, we propose a new precoding design that minimizes the transmit power while guaranteeing the quality of service $(\mathrm{QoS})$ and energy harvesting constraints for generic phase shift keying modulated signals. The QoS constraints are modified to accommodate constructive interference. We derive a sub-optimal solution and a local optimum solution to the precoding optimization problem. The proposed precoding reduces the transmit power compared to conventional schemes, by adapting the constraints to accommodate constructive interference as a source of useful signal power. Our simulation results show significant power savings with the proposed data-aided precoding compared to the conventional precoding.
\end{abstract}

\section{INTRODUCTION}

Recently simultaneous wireless information and energy transfer via the radio frequency energy harvesting $(\mathrm{EH})$ technology has emerged as a new solution for sustainable wireless network operation. In a multiuser scenario, interference signals provide a source for energy harvesting. On the other hand, there have been some efforts in exploiting constructive interference (CI) to improve the users' quality-of-service (QoS) and save the transmit power. This paper aims to exploit both data and channel state information (CSI) at the transmitter to facilitate the information and energy transfer in a multipleinput single-output (MISO) broadcast channel. Below we give brief literature review on simultaneous wireless information and energy transfer and data-aided precoding.

The fundamental concept of simultaneous wireless transmission of energy and information is introduced in [1] from an information theoretic standpoint. In [2] the authors discuss two practical receiver structures for simultaneous wireless information and energy transfer termed as "time switching" (TS) and "power splitting" (PS), to separate the received signal for decoding information and harvesting energy, respectively. Furthermore, multi-antenna technology can greatly facilitate the information and energy transfer. The optimal precoding design for energy and information transfer in a MISO broadcast channel is studied in [3]. The achievable information and energy transmission trade-offs are investigated in [4] for a 2-user multiple-input multiple-output (MIMO) interference channel (IC), based on different combinations of receiver modes. Joint information and energy transfer is studied for a general K-user MISO IC in [5] based on PS receivers.

Regarding the precoding design for a broadcast channel, the conventional view of data is that they are totally random and independent, and precoding design is independent of data. The proposed precoding in this paper is based on the viewpoint that data could be incorporated into the precoding design to better exploit the interference, which is first introduced in [6], [7], [8] where analytical interference classification criteria and low-complexity precoders based on channel inversion where derived. The analysis showed with the knowledge of both CSI and data at the base station (BS), some interfering data could contribute to the detection of the desired symbol and thus are classified as CI.

In this work we aim to optimize data-aided precoding design in a MISO broadcast channel with PS receivers by exploiting constructive interference as a useful source for both signal and energy transfer. We study the problem of transmit power minimization for guaranteeing both the signal-to-interferenceplus-noise ratio (SINR) and $\mathrm{EH}$ constraints. The problem has an interesting interpretation of multicast formulation. We propose a sub-optimal solution and a local optimum solution for the precoding design. Our results show that compared to the conventional precoding, the proposed data-aided precoding leads to 5-7 dB of power saving in high signal-to-noise ratio (SNR) region.

Notation: We use the upper case boldface letters for matrices and lower case boldface letters for vectors. $(\cdot)^{*}$ and $(\cdot)^{T}$ denote the conjugate and transpose, respectively. $\|\cdot\|$ stands for the Frobenius norm. A complex Gaussian random vector variable $\mathbf{z}$ with mean $\boldsymbol{\mu}$ and variance variance $\boldsymbol{\Sigma}$ is represented as $\mathbf{z} \sim \mathcal{C N}(\boldsymbol{\mu}, \boldsymbol{\Sigma}) . \mathbb{E}\{\cdot\}$ denotes the expectation. 


\section{System Model}

Consider a Gaussian MISO broadcast channel where an $\mathrm{N}$ antenna BS transmits both signals and energy to $K$ singleantenna users. For user $i$, its channel vector, precoding vector, received noise, data, SINR and EH constraints are denoted as $\mathbf{h}_{i}^{T}, \mathbf{t}_{i}, n_{i}, d_{i}, \Gamma_{i}, E_{i}$, respectively. The phase shift keying (PSK) modulated symbol can be expressed as $d_{i}=d e^{j \phi_{i}}$ where $d$ denotes the constant amplitude and $\phi_{i}$ is the phase. Without loss of generality, we assume $d=1$. The total transmit power is

$$
P_{T}=\mathbb{E}\left\{\left\|\sum_{k=1}^{K} \mathbf{t}_{k} d_{k}\right\|^{2}\right\} .
$$

$n_{i} \sim \mathcal{C N}\left(0, N_{0}\right)$ is the additive white Gaussian noise (AWGN). All wireless links exhibit independent frequency non-selective Rayleigh block fading. The received signal at user $i$ is

$$
y_{i}=\mathbf{h}_{i}^{T} \sum_{k=1}^{K} \mathbf{t}_{k} d_{k}+n_{i} .
$$

To decode the information and harvest RF energy at the receiver side, the practical PS technique [2] is used. Specifically, the receiver splits the RF signal into two parts: one for information decoding and the other for energy harvesting, with relative power of $\rho_{i}$ and $1-\rho_{i}$, respectively.

The signal for information decoding is expressed as

$$
\begin{aligned}
\tilde{y}_{i} & =\sqrt{\rho_{i}} y_{i}+\tilde{n}_{i} \\
& =\sqrt{\rho_{i}} \mathbf{h}_{i}^{T} \sum_{k=1}^{K} \mathbf{t}_{k} d_{k}+\sqrt{\rho_{i}} n_{i}+\tilde{n}_{i},
\end{aligned}
$$

where $\tilde{n}_{i} \sim \mathcal{C N}\left(0, N_{C}\right)$ is the complex AWGN introduced in the RF to baseband conversion in the decoding process, which is independent of $n_{i}$.

The signal for energy harvesting is

$$
\bar{y}_{i}=\sqrt{1-\rho_{i}} y_{i}=\sqrt{1-\rho_{i}}\left(\mathbf{h}_{i}^{T} \sum_{k=1}^{K} \mathbf{t}_{k} d_{k}+n_{i}\right)
$$

with average power

$$
P_{i}=\left(1-\rho_{i}\right) \mathbb{E}\left\{\left|\mathbf{h}_{i}^{T} \sum_{k=1}^{K} \mathbf{t}_{k} d_{k}+n_{i}\right|^{2}\right\} .
$$

The problem of interest is to minimize the total transmit power $P_{T}$ in (1) subject to QoS (i.e., SINR) constraints $\left\{\gamma_{i}\right\}$ and energy harvesting constraints $\left\{E_{i}\right\}$, respectively. This will be achieved by optimizing beamforming design, power allocation and splitting, as well as CI.

In the following, we first review the conventional precoding design then we introduce the proposed approach based on constructive interference.

\section{Review: Conventional Precoding}

In conventional MISO downlink precoding, users' data are independent to each other, i.e., $\mathbb{E}\left(d_{i}^{*} d_{j}\right)=0, \forall j \neq i$. In this case, the transmit power in (1) becomes

$$
P_{T}=\sum_{i=1}^{K}\left\|\mathbf{t}_{i}\right\|^{2}
$$

Based on the signal model (3) for information decoding, the received SINR for user $i$ is given by

$$
\Gamma_{i}^{c o n}=\frac{\left|\mathbf{h}_{i}^{T} \mathbf{t}_{i}\right|^{2}}{\sum_{j=1, j \neq i}^{K}\left|\mathbf{h}_{i}^{T} \mathbf{t}_{j}\right|^{2}+N_{0}+\frac{N_{C}}{\rho_{i}}} .
$$

The harvested energy can be derived from (5) as

$$
P_{i}^{c o n}=\left(1-\rho_{i}\right)\left(\sum_{k=1}^{K}\left|\mathbf{h}_{i}^{T} \mathbf{t}_{k}\right|^{2}+N_{0}\right) .
$$

Consequently, the power minimization problem with both QoS and $\mathrm{EH}$ constraints can be formulated as

$$
\begin{aligned}
\min _{\left\{\mathbf{t}_{i}, \rho_{i}\right\}} & \sum_{i=1}^{K}\left\|\mathbf{t}_{i}\right\|^{2} \\
\text { s.t. } & \Gamma_{i}^{c o n} \geq \gamma_{i}, \\
& P_{i}^{c o n} \geq E_{i}, \\
& 0<\rho_{i}<1, \forall i .
\end{aligned}
$$

It is easy to see that formulation $(8)$ is non-convex and hence challenging to solve. In our previous work [5], we have used semidefinite programming relaxation to tackle it and we show that the relaxation is tight for 2-user and 3-user MISO IC. This result is extended for the general MISO downlink in [3].

\section{Proposed Precoding with constructive INTERFERENCE}

\section{A. Problem Formulation}

Traditional precoding approaches treat users' data as totally random and independent information streams, however, for the PSK signalling considered, it is clear that each PSK symbol is merely a rotated version of another, i.e., $d_{j}=d_{i} e^{j\left(\phi_{j}-\phi_{i}\right)}$, therefore one user's data do not always generate harmful interference to others. With the knowledge of both the instantaneous CSI and the data symbols at the BS, the received interference can be classified to be constructive or destructive. In brief, while destructive interference performance, CI moves the received symbols away from the decision thresholds of the constellation and thus improves the detection. We refer the readers to [6], [7], [8] for further details. The main idea of the proposed precoding is to exploit the CI for both information decoding and energy harvesting.

The received signal at user $i$ in (2) can be rewritten as

$$
\begin{aligned}
y_{i} & =\mathbf{h}_{i}^{T} \sum_{k=1}^{K} \mathbf{t}_{k} d_{k}+n_{i} \\
& =\mathbf{h}_{i}^{T} \sum_{k=1}^{K} \mathbf{t}_{k} e^{j\left(\phi_{k}-\phi_{i}\right)} d_{i}+n_{i} .
\end{aligned}
$$




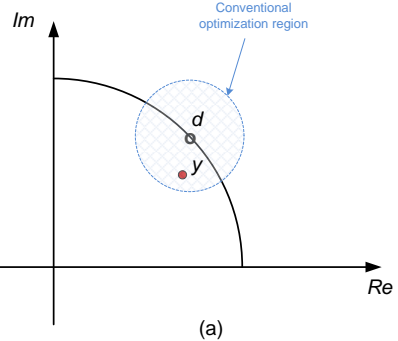

(a)

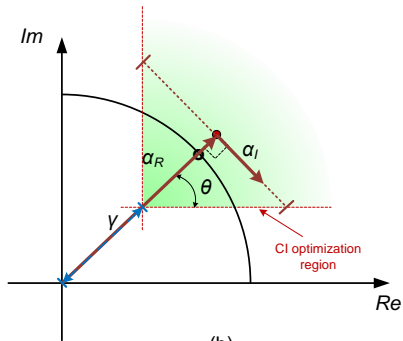

(b)

Fig. 1. Illustration of constructive interference for information decoding, QPSK example.

The information decoding part can be written as

$$
\sqrt{\rho_{i}} y_{i}+\tilde{n}_{i}=\sqrt{\rho_{i}} \mathbf{h}_{i}^{T} \sum_{k=1}^{K} \mathbf{t}_{k} e^{j\left(\phi_{k}-\phi_{i}\right)} d_{i}+\sqrt{\rho_{i}} n_{i}+\tilde{n}_{i} .
$$

We illustrate the derivation of the SINR constraint for the example of QPSK in Fig. 11. Here, Fig. 11.a) represents the conventional optimization region and Fig. 1 (b) shows the proposed optimization region. We have used the definitions $\alpha_{r}=\operatorname{Re}\left(\mathbf{h}_{i}^{T} \sum_{k=1}^{K} \mathbf{t}_{k} e^{j\left(\phi_{k}-\phi_{i}\right)}\right), \alpha_{i}=$ $\operatorname{Im}\left(\mathbf{h}_{i}^{T} \sum_{k=1}^{K} \mathbf{t}_{k} e^{j\left(\phi_{k}-\phi_{i}\right)}\right)$ and $\gamma=\sqrt{\Gamma_{i}\left(N_{0}+\frac{N_{C}}{\rho_{i}}\right)}$, where $\operatorname{Re}(x)$ and $\operatorname{Im}(x)$ denote the real part and imaginary part of $x$, respectively. Clearly, $\alpha_{r}$ represents the amplification of the received constellation point due to the CI and $\alpha_{i}$ gives the angle shift from the original constellation point. In conventional precoding optimization, $\alpha_{r}$ and $\alpha_{i}$ are constrained such that the received symbol is contained within a circle (denoted by the dashed circle) around the nominal constellation point, so that the interference caused by the other symbols is limited. In contrast to this, the concept of CI is exploited to allow a relaxation of $\alpha_{r}$ and $\alpha_{i}$ for all transmit symbols, under the condition that the interference caused is constructive, lying in the green shaded sector in the diagram [7]. It can be seen that $\alpha_{r}$ and $\alpha_{i}$ are allowed to grow infinitely, as long as their ratio is such that the received symbol is contained within the constructive area of the constellation, i.e. the distances from the decision thresholds, as set by the SNR constraints $\gamma$, are not violated. It can be seen that the angle of interference need not to be strictly aligned with the angle of the useful signal, as long as it falls within the constructive area of the constellation. For a given modulation order $M$ the maximum angle shift in the $\mathrm{CI}$ area is given by $\theta=\pi / M$. Using basic geometry we arrive at the SINR constraint expressed as

$$
\left|\alpha_{i}\right| \leq\left(\alpha_{r}-\gamma\right) \tan \theta,
$$

which is expanded as

$$
\begin{aligned}
& \left|\operatorname{Im}\left(\mathbf{h}_{i}^{T} \sum_{k=1}^{K} \mathbf{t}_{k} e^{j\left(\phi_{k}-\phi_{i}\right)}\right)\right| \leq \\
& \left(\operatorname{Re}\left(\mathbf{h}_{i}^{T} \sum_{k=1}^{K} \mathbf{t}_{k} e^{j\left(\phi_{k}-\phi_{i}\right)}\right)-\sqrt{\Gamma_{i}\left(N_{0}+\frac{N_{C}}{\rho_{i}}\right)}\right) \tan \theta .
\end{aligned}
$$

The harvested energy and the total transmit power can be derived based on (1) and (5), respectively, as $P_{i}=$ $\left|\mathbf{h}_{i}^{T} \sum_{k=1}^{K} \mathbf{t}_{k} e^{j\left(\phi_{k}-\phi_{i}\right)}\right|^{2}$ and $P_{T}=\left\|\sum_{k=1}^{K} \mathbf{t}_{k} e^{j\left(\phi_{k}-\phi_{i}\right)}\right\|^{2}$. Therefore, the power minimization problem subjective to both SINR and EH constraints with the aid of the CI can be formulated as

$$
\begin{aligned}
\min _{\left\{\mathbf{t}_{i}, \rho_{k}\right\}} & \left\|\sum_{k=1}^{K} \mathbf{t}_{k} e^{j\left(\phi_{k}-\phi_{i}\right)}\right\|^{2} \\
\text { s.t. } & {[12), } \\
& \left|\mathbf{h}_{i}^{T} \sum_{k=1}^{K} \mathbf{t}_{k} e^{j\left(\phi_{k}-\phi_{i}\right)}\right| \geq \sqrt{\frac{E_{i}}{1-\rho_{i}},} \\
& 0<\rho_{i}<1, \forall i .
\end{aligned}
$$

By defining $\tilde{\mathbf{h}}_{i}=\mathbf{h}_{i} e^{j\left(\phi_{1}-\phi_{i}\right)}$ and $\mathbf{w} \triangleq \sum_{k=1}^{K} \mathbf{t}_{k} e^{j\left(\phi_{k}-\phi_{1}\right)}$, we can write (13) equivalently as

$$
\begin{aligned}
\min _{\left\{\mathbf{w}, \rho_{i}\right\}} & \|\mathbf{w}\|^{2} \\
\text { s.t. } \quad & \left|\operatorname{Im}\left(\tilde{\mathbf{h}}_{i}^{T} \mathbf{w}\right)\right| \leq \\
& \left(\operatorname{Re}\left(\tilde{\mathbf{h}}_{i}^{T} \mathbf{w}\right)-\sqrt{\Gamma_{i}\left(N_{0}+\frac{N_{C}}{\rho_{k}}\right)}\right) \tan \theta \\
& \left|\tilde{\mathbf{h}}_{i}^{T} \mathbf{w}\right|^{2} \geq \frac{E_{i}}{1-\rho_{i}} \\
& 0<\rho_{i}<1, \forall i .
\end{aligned}
$$

Although the reformulation (14) seems a trivial step, it indicates that the original broadcast channel now reduces to a virtual multicast channel with common message to all users [9].

The problem (14) is nontrivial to solve because of the nonconvex constraint $\left|\tilde{\mathbf{h}}_{i}^{T} \mathbf{w}\right|^{2} \geq \frac{E_{i}}{1-\rho_{i}}$. The rest of this section is devoted to solving the multicast problem (14).

\section{B. A Sub-optimal Solution}

We first tackle the nonconvex constraint $\left|\tilde{\mathbf{h}}_{i}^{T} \mathbf{w}\right|^{2} \geq \frac{E_{i}}{1-\rho_{i}}$ by adding a new constraint $\operatorname{Im}\left(\tilde{\mathbf{h}}_{i}^{T} \mathbf{w}\right)=0$ then find a suboptimal solution. With this new constraint, the problem (14) becomes

$$
\begin{aligned}
\min _{\left\{\mathbf{w}, \rho_{i}\right\}} & \|\mathbf{w}\|^{2} \\
\text { s.t. } & \operatorname{Im}\left(\tilde{\mathbf{h}}_{i}^{T} \mathbf{w}\right)=0, \\
& \operatorname{Re}\left(\tilde{\mathbf{h}}_{i}^{T} \mathbf{w}\right) \geq \sqrt{\Gamma_{i}\left(N_{0}+\frac{N_{C}}{\rho_{i}}\right)}, \\
& \operatorname{Re}\left(\tilde{\mathbf{h}}_{i}^{T} \mathbf{w}\right) \geq \sqrt{\frac{E_{i}}{1-\rho_{i}}}, \\
& 0<\rho_{i}<1, \forall i .
\end{aligned}
$$

It is not difficult to verify that both $\sqrt{\Gamma_{i}\left(N_{0}+\frac{N_{C}}{\rho_{i}}\right)}$ and $\sqrt{\frac{E_{i}}{1-\rho_{i}}}$ are convex functions in $\rho_{i}$, so the above problem is 
convex. However, it can be further simplified. Observe that at the optimum, both constraints (17) and 18 can be satisfied with equality, i.e.,

$$
\Gamma_{i}\left(N_{0}+\frac{N_{C}}{\rho_{i}}\right)=\frac{E_{i}}{1-\rho_{i}}, \forall i,
$$

from which we can solve

$$
\rho_{i}^{*}=\frac{-B-\sqrt{B^{2}-4 A C}}{2 A},
$$

where $A=-\Gamma_{i} N_{0}, B=\Gamma_{i} N_{0}-E_{i}-\Gamma_{i} N_{C}, C=\Gamma_{i} N_{C} \cdot \rho_{i}^{*}$ is independent of $\mathbf{w}$. With $\rho^{*}$, the resulting problem is written as

$$
\begin{array}{ll}
\min _{\{\mathbf{w}\}} & \|\mathbf{w}\|^{2} \\
\text { s.t. } & \operatorname{Im}\left(\tilde{\mathbf{h}}_{i}^{T} \mathbf{w}\right)=0, \\
& \operatorname{Re}\left(\tilde{\mathbf{h}}_{i}^{T} \mathbf{w}\right) \geq \sqrt{\Gamma_{i}\left(N_{0}+\frac{N_{C}}{\rho_{i}^{*}}\right)}, \forall i .
\end{array}
$$

This is a quadratic problem with linear constraints in w, therefore easy to solve.

\section{The Proposed DC Algorithm}

Here we proposed another approach to tackle the nonconvex constraint (15) $\left|\tilde{\mathbf{h}}_{i}^{T} \mathbf{w}\right|^{2} \geq \frac{E_{i}}{1-\rho_{i}}$ using difference of convex optimization (DC) and successive convexification. This method will produce a local optimum solution which is normally better than the above sub-optimal solution.

To illustrate the idea, we define $f(\mathbf{v}) \triangleq\left|v_{1}\right|^{2}+\left|v_{2}\right|^{2}, v_{1} \triangleq$ $\operatorname{Re}\left(\tilde{\mathbf{h}}^{T} \mathbf{w}\right), v_{2} \triangleq \operatorname{Im}\left(\tilde{\mathbf{h}}^{T} \mathbf{w}\right)$. For convenience, we have dropped the user index. Then the linear approximation of $f(\mathbf{w})$ around the point $\mathbf{p}$ can be expressed as

$$
\begin{aligned}
f(\mathbf{v}) & =f(\mathbf{p})+\left(\left.\frac{\partial f(\mathbf{v})}{\partial \mathbf{v}}\right|_{\mathbf{v}=\mathbf{p}}\right)^{T}(\mathbf{v}-\mathbf{p}) \\
& =f(\mathbf{p})+2 \mathbf{p}^{T}(\mathbf{v}-\mathbf{p}) .
\end{aligned}
$$

Based on the above approximation, we propose the following algorithm to solve the problem (14).

The Proposed DC Algorithm:

1) Initialize $\mathbf{p}^{0}$.

2) At the iteration $k$, solve the problem

$$
\begin{aligned}
& \min _{\left\{\mathbf{w}, \rho_{i}\right\}}\|\mathbf{w}\|^{2} \\
& \text { s.t. }\left|\operatorname{Im}\left(\tilde{\mathbf{h}}_{i}^{T} \mathbf{w}\right)\right| \\
& \leq\left(\operatorname{Re}\left(\tilde{\mathbf{h}}_{i}^{T} \mathbf{w}\right)-\sqrt{\Gamma_{i}\left(N_{0}+\frac{N_{C}}{\rho_{i}}\right)}\right) \tan \theta, \quad(24) \\
& \left\|\mathbf{p}_{i}^{(k)}\right\|^{2}+2 \sum_{b=1}^{2}\left[\mathbf{p}_{i}^{(k)}\right]_{b}\left(\left[\mathbf{v}_{i}\right]_{b}-\left[\mathbf{p}^{(k)}\right]_{b}\right) \geq \frac{E_{i}}{1-\rho_{i}}, \\
& {\left[\mathbf{v}_{i}\right]_{1}=\operatorname{Re}\left(\tilde{\mathbf{h}}_{i}^{T} \mathbf{w}\right),\left[\mathbf{v}_{i}\right]_{2}=\operatorname{Im}\left(\tilde{\mathbf{h}}_{i}^{T} \mathbf{w}\right),} \\
& 0<\rho_{i}<1, \forall i .
\end{aligned}
$$

3) Update $\mathbf{p}^{(k+1)}=\left[\operatorname{Re}\left(\tilde{\mathbf{h}}_{i}^{T} \mathbf{w}\right) ; \quad \operatorname{Im}\left(\tilde{\mathbf{h}}_{i}^{T} \mathbf{w}\right)\right]^{T}$ until convergence.

The problem 23] is recognized as a convex problem and can be optimally solved. The proposed DC algorithm is proved to converge to a local optimum and it has been widely used in signal processing for communications [10].

There is one practical difficulty remaining. In the constraint 24), although $\sqrt{\Gamma_{i}\left(N_{0}+\frac{N_{C}}{\rho_{i}}\right)}$ is a convex function in $\rho_{i}$, it does not follow any composition rule therefore it is not recognized to be convex by most numerical solvers such as CVX [11]. In order to use CVX to solve it, we need the following procedures to explicitly show that the constraint $\sqrt{N_{0}+\frac{N_{C}}{\rho}} \leq g$ is convex where $g$ is a variable. For convenience, we have dropped the user index and the constant $\Gamma_{i}$. The key step is to introduce a new variable $u$ and rewrite the constraint $\sqrt{N_{0}+\frac{N_{C}}{\rho}} \leq g$ as

$$
\left\{\begin{array}{l}
\sqrt{N_{0}+u^{2}} \leq g, \\
\frac{N_{C}}{\rho} \leq u^{2} \Leftrightarrow \frac{\sqrt{N_{C}}}{u} \leq \sqrt{\rho} .
\end{array}\right.
$$

It is easy to check that both of the above constraints in 25 are convex jointly in $(\rho, u, g)$ using the composition rules [12], thus they can all be recognized by CVX.

\section{Finding a Feasible Solution}

The remaining issue is how to find a feasible solution for the proposed DC algorithm. This can be achieved by solving the problem (14) without the EH constraint (15) for arbitrary $\rho_{i}\left(0<\rho_{i}<1\right)$, which is a second-order cone program problem and can be optimally and efficiently solved. If the obtained solution satisfies the EH constraint $\left|\tilde{\mathbf{h}}_{i}^{T} \mathbf{w}\right|^{2} \geq \frac{E_{i}}{1-\rho_{i}}$, then a feasible solution is found; otherwise, increase the power w such that the constraint $\left|\tilde{\mathbf{h}}_{i}^{T} \mathbf{w}\right|^{2} \geq \frac{E_{i}}{1-\rho_{i}}, \forall i$, is satisfied. This power amplification will not affect the SINR constraint.

\section{NumERICAL RESULTS}

In this section, we numerically assess the performance of the proposed data-aided CI precoding scheme. Channels are independent and experience frequency flat Rayleigh fading with zero mean and unit variance. We compare the required BS transmit power of the proposed DC solution, the sub-optimal solution, and the conventional solution without considering the CI described in Section III . Unless otherwise specified, QPSK is the default modulation scheme and all users have the same SINR and EH constraints. $K=N_{t}=4$ and $N_{0}=N_{1}=1$ are assumed. All figures are produced by averaging the results of 100 channel instances.

Fig. 2 depicts the transmit power of the different schemes versus the SINR requirement when the minimum required $\mathrm{EH}$ is $10 \mathrm{~dB}$. The transmit power with only SINR constraints (no $\mathrm{EH}$ constraints) for the conventional and CI based schemes is also shown for comparison. We can see that when the SINR constraint is below $5 \mathrm{~dB}$, the conventional solution outperforms the proposed CI based solution. However, when the SINR constraint is greater than $10 \mathrm{~dB}$, there is a sharp 
rise of the transmit power for the conventional scheme; the proposed DC solution and the sub-optimal solution achieve power reduction of $7 \mathrm{~dB}$ and $5 \mathrm{~dB}$, respectively. This can be explained by the fact that when SINR is low, the required transmit power is also low. Consequently, there is not sufficient interference among users to exploit, which is also disadvantageous for the proposed CI scheme. It is also seen that as SINR increases, the transmit power converges to the solution with only SINR constraint and this is because in the high SINR region, there is sufficiently high interference thus EH constraints are automatically satisfied.

In Fig. 3, we compare the transmit power against the EH requirement when the minimum required SINR is $20 \mathrm{~dB}$. In this case, the interference among users is high and therefore the proposed CI based solutions outperform the conventional solution. Similar performance gain in terms of power reduction as Fig. 2 is observed consistently in the whole EH region.

Finally the effect of the number of transmit antennas is illustrated in Fig. 4, when both SINR and EH constraints are $20 \mathrm{~dB}$. As can be seen, the benefit of the proposed CI solution diminishes compared to the conventional scheme as the number of transmit antennas increases. When the number of transmit antennas is greater than 8 , the performance gap among different schemes is negligible. One possible reason for this observation is that when the number of transmit antennas is large, users' channels tend to be more orthogonal to each other resulting in low interference.

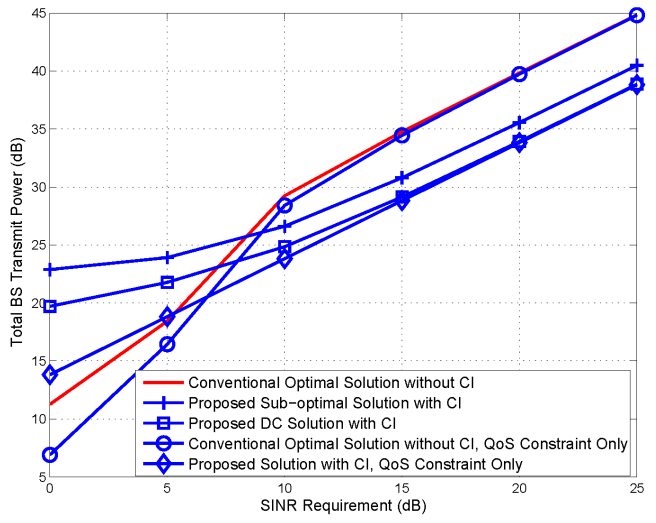

Fig. 2. Transmit power vs. SINR constraints, $E_{i}=10 \mathrm{~dB}$.

\section{CONCLUSions}

In this paper, we exploited the interference in MISO downlink to boost the performance for both information transfer and energy harvesting. Both sub-optimal and DC based solutions are derived to achieve the precoder design. Numerical results demonstrate that the proposed schemes significantly reduce transmit power by 5-7 dB compared to the conventional precoding design in the high SNR region.

\section{REFERENCES}

[1] P. Grover and A. Sahai, "Shannon meets Tesla: wireless information and power transfer," in Proc. IEEE Int. Symp. Inf. Theory, Austin, USA, June 2010, pp. 2363-2367.

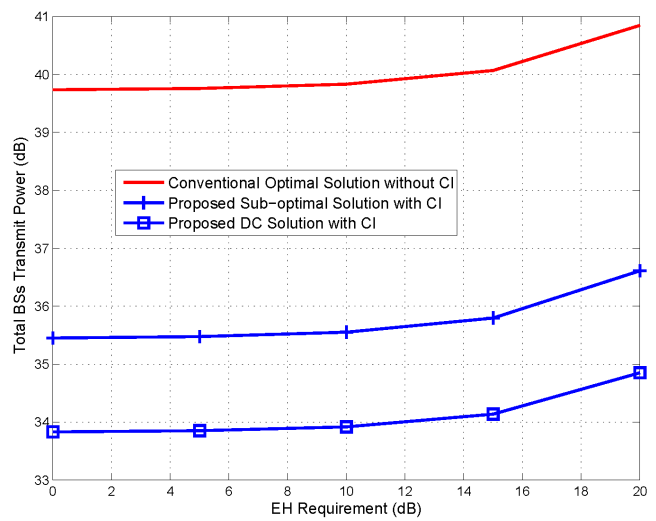

Fig. 3. Transmit power vs. EH constraint constraints, $\Gamma_{i}=20 \mathrm{~dB}$.

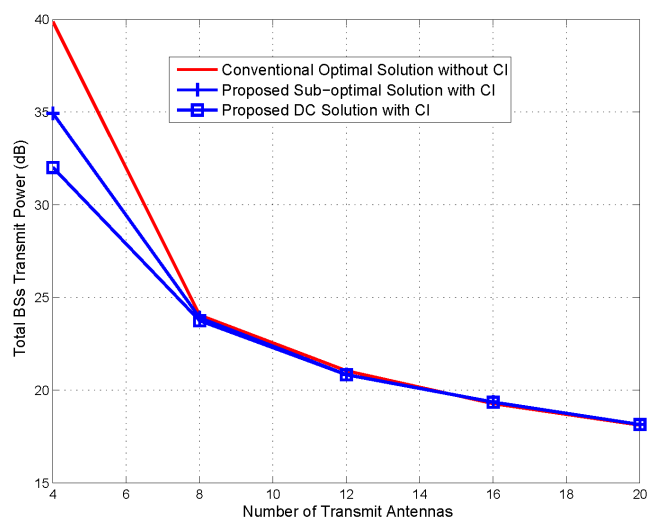

Fig. 4. The effect of the number of transmit antennas, $\Gamma_{i}=E_{i}=20 \mathrm{~dB}$.

[2] R. Zhang and C. K. Ho, "MIMO broadcasting for simultaneous wireless information and power transfer," IEEE Trans. Wireless Commun., vol. 12, no. 5, pp. 1989-2001, May 2013.

[3] Q. Shi, L. Liu, W. Xu, and R. Zhang, "Joint transmit beamforming and receive power splitting for MISO SWIPT systems," IEEE Trans. Wireless Commun., vol, 13, no. 6, pp. 3269-3280, June 2014.

[4] J. Park and B. Clerckx, "Joint wireless information and energy transfer in a two-user MIMO interference channel," IEEE Trans. Wireless Commun., vol. 12, no. 8, pp. 4210-4221, Aug. 2013.

[5] S. Timotheou, I. Krikidis, G. Zheng, and B. Ottersten, "Beamforming for MISO interference channels with QoS and RF energy transfer," IEEE Trans. Wireless Commun., vol. 13, no. 5, pp. 2646-2658, May 2014.

[6] C. Masouros, E. Alsusa, "Dynamic linear precoding for the exploitation of known interference in MIMO broadcast systems," IEEE Trans. Wireless Comms., vol. 8, no. 3, pp. 1396-1404, Mar. 2009.

[7] C. Masouros, "Correlation rotation linear precoding for MIMO broadcast communications", IEEE Trans. Sig. Proc., vol. 59, no. 1, pp. 252-262, Jan. 2011.

[8] C. Masouros, T. Ratnarajah, M. Sellathurai, C. Papadias, A. Shukla, "Known interference in wireless communications: a limiting factor or a potential source of green signal power?," IEEE Comms. Mag., vol. 51, no. 10, pp. 162-171, Oct. 2013.

[9] N. D. Sidiropoulos, T. N. Davidson, Z. Q. Luo, "Transmit beamforming for physical-layer multicasting," IEEE Trans. Signal Proc., vol. 54, no. 6, pp. 2239-2251, June 2006.

[10] L. Tran, M. Hanif, and M. Juntti, "A conic quadratic programming approach to physical layer multicasting for large-scale antenna arrays," IEEE Sig. Proc. Lett., vol. 21, no. 1, pp. 114-117, Jan. 2014.

[11] CVX Research Inc., "CVX: Matlab software for disciplined convex programming," http://cvxr.com/cvx. Sept. 2012.

[12] S. Boyd and L. Vandenberghe, "Convex Optimization," Cambridge University Press, 2004. 